LA-UR- $95-2628$

$\operatorname{CONF}-950846--9$

Los Alamos National Laboratory is operated by the University of California for the United States Department of Energy under contract W-7405-ENG-36

TILE: NUMERICAL ANOMALIES MIMICKING PHYSICAL EFFECTS

AUTHOR(S): Ralph Menikoff, T-14

SUBMITED TO: $\quad$ APS Topical Conference on Shock Compression in Condensed Matter, August 13-18, 1995

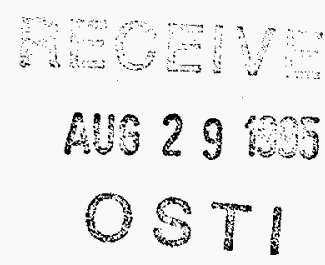

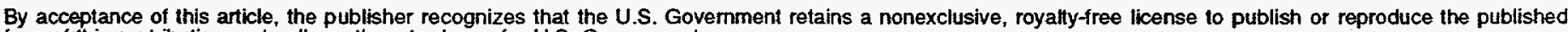
form of this contribution, or to allow others to do so, for U.S. Government purposes.

The Los Alamos National Laboratory requests that the publisher identify this article as work performed under the auspices of the U.S. Department of Energy.

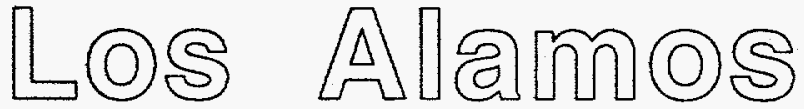

FORM NO. 836 R4 ST. NO. $26295 / 81$

\section{Los Alamos National Laboratory Los Alamos, New Mexico 87545}




\section{DISCLAIMER}

This report was prepared as an account of work sponsored by an agency of the United States Government. Neither the United States Government nor any agency thereof, nor any of their employees, make any warranty, express or implied, or assumes any legal liability or responsibility for the accuracy, completeness, or usefulness of any information, apparatus, product, or process disclosed, or represents that its use would not infringe privately owned rights. Reference herein to any specific commercial product, process, or service by trade name, trademark, manufacturer, or otherwise does not necessarily constitute or imply its endorsement, recommendation, or favoring by the United States Government or any agency thereof. The views and opinions of authors expressed herein do not necessarily state or reflect those of the United States Government or any agency thereof. 


\section{DISCLAIMER}

Portions of this document may be illegible in electronic image products. Images are produced from the best available original document. 


\title{
NUMERICAL ANOMALIES MIMICKING PHYSICAL EFFECTS
}

\author{
Ralph Menikoff
}

\author{
Theoretical Division, Los Alamos National Laboratory, Los Alamos, NM 87545
}

\begin{abstract}
Numerical simulations of flows with shock waves typically use finite-difference shock-capturing algorithms. These algorithms give a shock a numerical width in order to generate the entropy increase that must occur across a shock wave. For algorithms in conservation form, steady-state shock waves are insensitive to the numerical dissipation because of the Hugoniot jump conditions. However, localized numerical errors occur when shock waves interact. Examples are the "excess wall heating" in the Noh problem (shock reflected from rigid wall), errors when a shock impacts a material interface or an abrupt change in mesh spacing, and the start-up error from initializing a shock as a discontinuity. This class of anomalies can be explained by the entropy generation that occurs in the transient flow when a shock profile is formed or changed. The entropy error is localized spatially but under mesh refinement does not decrease in magnitude. Similar effects have been observed in shock tube experiments with partly dispersed shock waves. In this case, the shock has a physical width due to a relaxation process. An entropy anomaly from a transient shock interaction is inherent in the structure of the conservation equations for fluid flow. The anomaly can be expected to occur whenever heat conduction can be neglected and a shock wave has a non-zero width, whether the width is physical or numerical. Thus, the numerical anomaly from an artificial shock width mimics a real physical effect.
\end{abstract}

\section{INTRODUCTION}

Fluid flow is modeled by continuum equations which describe conservation of mass, momentum, and energy. When transport effects are neglected the fluid flow equations form a system of hyperbolic partial differential equations (PDEs)

$$
\frac{\partial}{\partial t}\left(\begin{array}{c}
\rho \\
\rho u \\
\rho E
\end{array}\right)+\frac{\partial}{\partial x}\left(\begin{array}{c}
\rho u \\
\rho u^{2}+P \\
\rho u(E+P V)
\end{array}\right)=\left(\begin{array}{l}
0 \\
0 \\
0
\end{array}\right),
$$

where $\rho, u$, and $e$ are the density, particle velocity, and specific internal energy. $E=\frac{1}{2} u^{2}+e$ is the total internal energy, $V=1 / \rho$ is the specific density, and $P(V, e)$ is the pressure.

For smooth flows entropy is convected along particle paths. When characteristics cross and smooth flow breaks down, discontinuous shock waves form. Both the physics being modeled and the mathematical stability of solutions to the PDEs require that entropy increases across a shock wave. Yet the PDEs contain no explicit dissipative terms. This has important consequences for numerical algorithms to simulate fluid flow.

Soon after computers first became available, it was discovered that straightforward finitedifference algorithms for the fluid equations become wildly unstable if a shock wave occurs in the flow. This led von Neumann and Richtmyer (1) to introduce an artificial viscosity in order to add numerical dissipation and stabilize the calculation. Their idea was to modify the PDEs 
such that (i) the correct state behind a shock is achieved, (ii) smooth flows are minimally affected, and (iii) the time step for an explicit algorithm is not unduly restricted. Artificial viscosity gives a shock wave a smooth profile which is analogous to the profile due to real viscosity. However, the numerical shock width is constrained by the grid resolution and is artificially large compared to the real physical shock width.

The numerical scheme meets its objectives because (i) the state behind a steady-state shock wave is determined by the Hugoniot jump conditions and is independent of the form of the dissipation, (ii) by choosing a non-linear form for the artificial viscosity, its dissipative effect is limited to regions with large gradients, i.e., the shock profile, and (iii) by choosing the magnitude of the artificial viscosity only as large as is needed for stability, the shock profile is limited to a few cells and the time step restriction from the domain of dependence is comparable both for the hyperbolic equations (without viscosity) and the parabolic equations (with viscosity). These heuristic arguments suggest that the solution to the modified equations (with viscosity) differs from that of the fluid equation (without viscosity) only in the vicinity of a shock front.

The idea of giving a shock front a profile of a few cells is the basis for "shock-capturing" algorithm. These algorithms have evolved to use approximate Riemann solvers in order to generate the necessary numerical dissipation for shock waves and to account properly for the flow of information along characteristics. Moreover, when the algorithms are written in conservation form, local errors at the shock front tend not to accumulate. Generally, shock-capturing algorithms give accurate solutions. However, calculated solutions sometimes display "glitches" or unexpected localized errors. Examples are the "excess wall heating" in the Noh problem (2) of a strong shock reflected from rigid wall, errors when a shock impacts a material interface or an abrupt change in mesh spacing, and the start-up error from initializing a shock as a discontinuity.

This class of anomalies is an unavoidable side effect of the numerical shock profile when heat conduction is neglected. Similar physical effects occur for partly dispersed shock waves (3). In the physical case, the wave width can be due to a relaxation process or a chemical reaction. In contrast to a steady-state wave, the transient entropy generation when a shock profile is abruptly formed or changed is not determined by the Hugoniot jump conditions and can depend on the dissipation mechanism. This motivates us to consider the interaction of a shock wave with either another shock wave or a contact.

The analysis of a shock interaction is based on the variation of the conserved quantities within a shock profile given by Menikoff (4). The key points are that (i) the conserved quantities within a profile are a function of shock strength, (ii) there is a difference in the conserved quantities contained within the shock profiles of the ingoing and outgoing waves, and (iii) outside a shock profile the entropy generation is negligible. As a consequence, there must be an entropy anomaly in the interaction region. Moreover, without heat conduction, the entropy anomaly is frozen into the particle paths and does not diffuse away.

Since the argument is based solely on the conservation laws, it applies both to the continuum PDEs and to their finite-difference approximations in conservation form. An entropy anomaly can be expected whenever shock waves have a non-zero width and interact. It does not matter whether the shock width is physical or numerical. Thus, the numerical anomaly from an artificial shock width mimics a real physical effect. Moreover, under mesh refinement the spatial extent of the anomaly shrinks but the magnitude of the anomaly remains the same. 


\section{CONSERVED QUANTITIES}

Due to the Hugoniot jump conditions, the states ahead and behind a steady-state shock wave is independent of the shock profile. Typically, the length scale associated with the wave width is very small and can be neglected. However, the variation of conserved quantities within a shock profile can lead to anomalies. The first step in comparing waves with different profiles is to define the shock position.

Let the subscripts $a$ and $b$ denote the states on the shock Hugoniot ahead and behind the wave, and let $\sigma$ denote the wave speed. We define the shock position $x_{s}$ of a partly dispersed shock to match the mass of the corresponding discontinuous shock. For a right facing wave,

$$
\int_{x<x_{s}} d x\left[\rho_{b}-\rho(x)\right]=\int_{x_{s}<x} d x\left[\rho(x)-\rho_{a}\right] .
$$

The mass flux in a steady-state wave, $\rho(\sigma-u)$, is constant throughout the profile. Consequently, matching the momentum

$\int_{x<x_{s}} d x\left[\rho_{b} u_{b}-\rho(x) u(x)\right]=\int_{x_{s}<x} d x\left[\rho(x) u(x)-\rho_{a} u_{a}\right]$. gives the same shock position.

The total energy is not a Galilean invariant. As a result, the shock position based on matching energy is frame dependent. Instead we consider the energy of a dispersed wave relative to the discontinuous shock

$$
\begin{aligned}
\delta \mathcal{E}=\int_{x<x_{b}} d x[\rho(x) & \left.E(x)-\rho_{b} E_{b}\right] \\
& +\int_{x_{s}<x} d x\left[\rho(x) E(x)-\rho_{a} E_{a}\right] .
\end{aligned}
$$

Provided that the shock position is chosen to match the mass and momentum, the relative energy is independent of frame. In general, $\delta \mathcal{E}$ depends on the form of dissipation (or numerical algorithm), the shock strength, and the material equation of state.

\section{NUMERICAL ANOMALIES}

In a transient, when a shock profile is formed or changed, the Hugoniot jump conditions do not apply. The variation of the conserved quantities within the shock profile leads to a localized entropy anomaly. Four common examples of this type of anomaly are described.

\section{Symmetric Shock Interaction}

Our first example is the symmetric collision of two shocks. Usually a shock interaction results in two outgoing waves and a contact. For a symmetric collision the contact is degenerate, i.e., of zero strength. Moreover, a symmetric shock interaction is equivalent to a shock reflecting from a rigid wall. In effect, there is one incoming shock and one outgoing shock.

Consider the reflection of a dispersed shock wave from a rigid wall. Suppose the state behind the outgoing wave is uniform. Then the position of the outgoing shock is uniquely determined by mass conservation. There is no constraint from total momentum since the wall can act as a momentum source. However, the relative energies of the shock profiles are not the same, $\delta \mathcal{E}_{\text {in }} \neq \delta \mathcal{E}_{\text {out }}$, because the incoming and outgoing waves have different shock strengths. Therefore, energy conservation implies a uniform state behind the outgoing wave is not possible.

In order that dissipation does not affect smooth flow, the asymptotic shock profile must be reached after the outgoing wave has propagated a distance on the order of the width of its profile. Moreover, the pressure and particle velocity behind the outgoing wave equilibrates to the solution of the Riemann problem for the hyperbolic equations on the same time scale as for a shock to equilibrate to its asymptotic profile. As a result, the difference between the relative energies of the incoming and outgoing 
shock profiles must show up in the interaction region in which the profiles of the incoming and outgoing waves overlap. Therefore, the transient change in a shock profile leads to an entropy anomaly. Without heat conduction, the entropy is frozen into the particle paths and the anomaly does not diffuse away. Because the particle velocity vanishes at the wall, a shock reflection results in an entropy boundary layer at the wall.

Shock tube experiments have been used to study rate processes in gases (3). When a relaxation process leads to partly dispersed shock waves, experiments have measured the entropy boundary layer that occurs when a shock reflects from a wall. Calculations that include the physical dissipative mechanism and resolve the shock profile also show an entropy boundary layer.

The same phenomenon occurs in the Noh problem used to test shock-capturing algorithms. The Noh problem is the initial value problem in which a cold ideal gas is given a velocity directed towards a rigid wall. With artificial viscosity for numerical dissipation, Noh (3) found that a numerical error occured at the wall in which a high energy is compensated for by a low density to give the correct pressure. Noh called this anomaly "excess wall heating." Since the error is associated with the transient dissipation when a shock profile is formed, it is more appropriate to consider the effect as a numerical entropy anomaly.

\section{Impedance Match}

A non-degenerate contact occurs for a nonsymmetric shock interaction. The difference in relative energy between the incoming and outgoing shock profiles can be taken up by smearing out the contact. Furthermore, the velocity of the contact is in general non-zero. Numerical advection in an Eulerian algorithm is a diffusive process. Its effect on entropy at a contact is qualitatively similar to heat conduction. Thus, the entropy anomaly from a shock interaction, or for the solution to a Riemann problem, can be greatly reduced by numerical diffusion.

When the contact is a material interface and the equations of state of the two materials are very different, then a numerical algorithm must minimize diffusion across a contact. As a second example, we consider an impedance match when a shock wave is incident on a material interface. In this case, the incoming waves are the incident shock and the contact, and there are two outgoing wave with the material interface serving as the contact.

Again we show that uniform states behind the outgoing waves are not possible. After the asymptotic shock profiles have formed, the solution has three degrees of freedom corresponding to the positions of the transmitted and reflected waves and the position of the contact. The conservation laws leads to four constraints: the mass of each material, total momentum, and total energy. This gives four conditions for three unknowns. Because of the Hugoniot jump conditions, the hyperbolic problem has a solution, namely; the solution to the Riemann problem with initial data corresponding to the time when the incident shock impacts the material interface. However, the difference in the relative energies between the incoming and outgoing shock profiles results in the overdetermined equations being incompatible. Therefore, the states behind the waves can not be uniform.

Again the outgoing shocks equilibrate rapidly to their equilibrium profiles and there is no dissipation away from the shock profiles. Therefore, the energy difference gives rise to an entropy anomaly in the transient region in which the wave profiles overlap. Hence, an impedance match results in an entropy anomaly at the material interface. 


\section{Abrupt Change Of Grid}

For a shock-capturing algorithm, the shock width is proportional to the grid spacing. Therefore, the relative energy of a shock profile scales with the grid spacing. The difference in relative energy between the incident and transmitted shocks causes an entropy anomaly at the interface where the grid changes. Thus, a shock traversing through an abrupt change in the grid spacing is qualitatively similar to an impedance match. The main difference is that a weak reflected wave occurs instead of a shock. The weak wave is discussed in more detail in the next subsection.

In particular, suppose a shock propagates from a coarse mesh to a fine mesh. Suppose the shock width is 3 cells. We neglect the fact that the CFL factor $c \Delta t / \Delta x$ is smaller for the coarse mesh and hence a shock is spread out over a larger number of cells on the coarse mesh. The rise time of the pressure profile, $\tau=3 \Delta x / \sigma$, is less for the fine mesh than for the coarse mesh. As a consequence, the transmitted shock propagates through at least $3\left(\Delta x_{c} / \Delta x_{f}\right)$ cells before its asymptotic profile is reached. Furthermore, since the rise time of the pressure driving the transmitted shock is greater than the rise time of the shock profile, the entropy generation in the cells before the asymptotic profile is reached can be expected to have a smaller value than for the steady-state shock

This example illustrates that the dissipation in a transient can be affected by the mesh and also by the algorithm.

\section{Shock Start Up Error}

Another common numerical glitch arises when a shock wave is initialized as a discontinuity. This can be viewed as a numerical Riemann problem. In general the solution to a Riemann problem results in three waves. In this case, the dominant wave is a shock. If the shock has a zero width then the other two waves would be degenerate. Because of the relative energy required to form the numerical shock profile, an entropy anomaly or smeared out contact occurs. In addition, a secondary weak wave of the opposite family arises.

The initial data is incompatible with a secondary shock or rarefaction wave. Instead an $\mathrm{N}$-wave, shock-rarefaction-shock, is formed. Numerical dissipation smears the $\mathrm{N}$-wave into a discrete diffusion wave (5). The difference between the shock profile and the discontinuous shock can be thought of as a localized perturbation on a uniform flow corresponding to the state behind the shock. A general property of hyperbolic PDEs is that the asymptotic solution to the perturbed problem consists of a superposition with one outgoing wave for each wave family (6). The next weaker wave after a shock is an $\mathrm{N}$-wave. Unlike a shock, an $\mathrm{N}$-wave spreads out and decays in time. The corresponding finite difference equations in conservation form have similar wave properties to the hyperbolic PDEs with the addition of viscosity.

\section{ADDITIONAL EFFECTS}

The non-zero wave width and the discretization in finite-difference shock-capturing algorithm leads to additional numerical anomalies. We briefly describe a few other of the important effects and give heuristic explanations for the anomalies.

As the shock position traverses through a cell, the discretization of the shock profile causes the conserved quantities within the profile to vary. This acts as a source of acoustic radiation. In addition, when a shock propagates at an angle to the mesh in multi-dimensions, the shock profile varies with position along the shock front. This is also a source of acoustic noise. An equivalent 
physical effect occurs when an instability causes the wave profile to oscillate. An example, is a galloping one-dimensional detonation wave (7).

For upwind schemes, the shock overtakes the acoustic wave ahead of the front. Usual the radiation behind the front has a short wavelength and high frequency. Since the mesh can not resolve these signals, numerical stability requires that the algorithm damp the down stream acoustic noise. However, for Eulerian algorithms and a. nearly stationary shock, the acoustic noise from the variation of the shock profile has a long wavelength and is indistinguishable from acoustic signals the mesh is supposed to resolve. Consequently, there is more numerical noise behind a nearly stationary shock than a shock that moves relative to the mesh. This example illustrates how the finite-difference discretization can break the Galilean invariance of the continuum equations.

When the shock front is curved, a non-zero shock width leads to a curvature effect on the propagation of a shock wave. One example of the curvature effect is observable in wind tunnel experiments of steady supersonic flow over a wedge. An attached oblique shock has a significant curvature near the tip of the wedge (3). Another important example is the diameter effect in a rate stick of explosive (detonation velocity versus diameter). The curvature effect is due to the competition within the reaction zone of a source term for the release of chemical energy and a geometric source term from the front curvature (8). The effect is proportional to the dimensionless product of the wave width and the front curvature. When the reaction zone of a detonation wave is not resolved, there is a numerical curvature effect which is mesh dependent and is artificially large due to the artificially large reaction zone width. The change in wave speed from the artificial length scale can have a significant effect on the global flow.
The global flow can be affected in two other cases. First, when a local error seeds a perturbation for a subsequent instability. Second, near a bifurcation of a two dimensional wave pattern. The transition between regular and Mach reflection is an example of a bifurcation. The threshold may be affected by dissipative mechanisms. In particular, the numerical dissipation of a particular algorithm could shift the threshold and thus affect the global flow.

Shock-capturing algorithms tacitly assume that the numerical solution is independent of sub-grid physical phenomena. However, we have shown that there are physical anomalies caused by the dissipative mechanisms that gives a shock wave a non-zero width. These physical effects can be used to understand common numerical error involving shock waves. The important question is when do these errors remain localized and when do they affect the global flow.

\section{ACKNOWLEDGMENT}

This work supported by U. S. Dept. of Energy. The author is indebted to Klaus Lackner for many stimulating discussions on the effect of shock width on wave propagation.

\section{REFERENCES}

1. von Neumann, J., Richtmyer, R. D., J. Appl. Phys. 21 232-237 (1950).

2. Noh, W. F., J. Comp. Phys. 72, 78-120 (1987).

3. Becker, E., Ann. Rev. of Fluid Mech. 4 155-194 (1972).

4. Menikoff, R., SIAM J. Sci. Comput. 15, 1227-1242 (1994).

5. Chern, I.-L., Mathematics of Computation 56, 107-118 (1991).

6. Liu, T.-P., Commun. Math. Phys. 68, 141-172 (1979).

7. Alpert, R. L., Toong, T.-Y., Astronautica Acta. 17, 539-560 (1972).

8. Menikoff, R., Lackner, K. S., Bukiet, B. G., Combustion and Flame, to appear (1995). 